\title{
Molecular Characterization of the GBA Gene in Patients from Southwest of Colombia with Gaucher Disease
}

Journal of Inborn Errors of Metabolism \& Screening 2021, Volume 9: e20200018 DOI: https://doi.org/10.1590/2326-4594JIEMS-2020-0018

\author{
Daniela Arturo Terranova ${ }^{1,4,6}$ (D), Lina Johanna Moreno Giraldo, 2,3,4,5,6,7 (D) \\ Henry Idrobo ${ }^{1,6,7}$ and José María Satizabal',2,4,5,6,7 $\mathbb{D}$
}

\begin{abstract}
Introduction: Gaucher's disease (GD) is an autosomal-recessive lysosomal storage disorder that results from hereditary deficiency of the acid glucocerebrosidase enzyme, encoded by the GBA gene necessary for the degradation of glucosylceramide. Objective: molecularly characterize the variants found in the GBA gene present in patients from the Southwest of Colombia with GD.

Material and methods: 19 patients were included in the study, clinically and enzymatically diagnosed with GD. A molecular analysis of the GBA gene was performed and the variants were subsequently searched in different population and clinical databases. A bioinformatic analysis was performed.

Results: The variants in the GBA gene reported were classified into: 14/19 homozygous patients, 4/19 compound heterozygote and $1 / 19$ heterozygous. The presence of 7 variants coding for 8 different genotypes was reported. Also the known mutations like Asn409Ser, p.Leu483Pro, p.Lys237Glu, p.Glu427Lys, and p.Arg535His were identified in these patients. The most frequent genotype was p. Asn409Ser / Asn409Ser (36\%). All the variants presented a pathogenic clinical significance.

Conclusion: The given study will make it possible to understand the susceptibility to GD in the population. This can help maintain the health quotient of the population through premarital counseling and therefore minimize the burden of disease among the population.
\end{abstract}

Keywords: Computational Biology, Gaucher Disease, GBA gene, Lysosomal Storage Diseases, Variants (DeCS).

\section{Introduction}

Gaucher disease (GD) (MIM \# 230800, 230900, 231000; CIE10 E75.2; ORPHA: 355) is one of the most frequent lysosomal storage disorders, with an inheritance of autosomal recessive type, caused by a mutation or variants in the GBA gene located in the chromosome 1 in a region designated q21. This disease is characterized by a partial or total deficiency of the enzyme beta-glucocerebrosidase (acid $\beta$-glucosidase) which is necessary to degrade a type of lipid known as glucosylceramide, which, as a consequence of the enzymatic deficit, it is accumulated in the body, predominantly in the liver, spleen and bone marrow.[1,2]

GD has a pan-ethnic distribution with cases described worldwide. In the Ashkenazi Jewish (AJ) population, incidence has been found $(1 / 1,000)$ and GD is not considered a rare disease. [3,4] Studies by Nalysnyk et al. (2017) found that the estimate of incidence at birth, in communities with mixed ancestry, varies from 1 case per 17,421 live births (LB) in Austria, and to 1 case per 256,410 LB in Canada.[5] Orphanet reports a prevalence in Europe of about 1 case per 100,000 population.[6]

\footnotetext{
${ }^{1}$ Universidad del Valle, Cali, Valle del Cauca, Colombia.

'Universidad Santiago de Cali, Valle del Cauca, Colombia.

${ }^{3}$ Universidad Libre, Cali, Valle del Cauca, Colombia.

${ }^{4}$ Universidad del Valle, Postgraduate in Biomedical Sciences, Cali, Valle Del Cauca, Colombia.

${ }^{5}$ Universidad del Valle, Faculty of Health, Emphasis on medical genetics, Cali, Colombia.

'Universidad del Valle, Congenital Metabolism Diseases Research Group, Category A Colciencias 2019, Cali, Colombia.

${ }^{7}$ Colombian Interest Group on Gaucher Disease, Cali, Colombia.
}

Received october 22, 2020, and in revised form april 5, 2021. Accepted for publication april 29, 2021.

\section{Corresponding Author:}

Daniela Arturo Terranova, E-mail: daniela.arturo@correounivalle.edu.co 
In Colombia, according to the Colombian Association of Patients with Lysosomal Storage Diseases - ACOPEL, by its Spanish initials -, the estimated prevalence of GD is 1 per 266,441 inhabitants. [7] The studies carried out by Uribe et al. (2013), in which 4,700 patients with suspected alterations in lysosomal metabolism were evaluated, reported 101 patients affected by GD.[8] In the last report made by Puentes-Tellez et al. (2020) in a Colombian study carried out in 32,940 individuals: $3,834(11.6 \%)$ evaluated with the classical methods (urine / serum / whole blood analysis) and 29,106 (88.4\%) evaluated in dried blood samples (since 2005), a total of 196 patients affected with GD were reported.[9] However, exact figures are not yet available on the incidence and prevalence of this disease, since, lack of knowledge, clinical heterogeneity, or difficult diagnosis, it is an under-diagnosed disease in Colombia, even though the GD is included in the list of 2,198 Orphan Diseases (OD) in the country according to Resolution 5265 of 2018.[7]

More than 460 pathogenic mutations have now been identified in $G B A$ gene but the most commonly occurring mutations like- p.Asn409Ser (p.Asn370Ser according to the old nomenclature), IVS2, 84GG, p.Leu483Pro (p.Leu444Pro, according to the old nomenclature) and c.84dupG (84GG) are responsible of approximately $96.5 \%$ of the GD in the AJ population in the Western Hemisphere, and approximately $50 \%-60 \%$ in non-Jewish populations. The mutation c. $1448 \mathrm{~T}>\mathrm{C}$ (Leu444Pro) is pan-ethnic.[1,10]

The low expression of GBA gene determines the heterogeneous clinical manifestations of the disease.[1-2] Currently, GD is classified into 3 basic types depending on the presence and severity of neurological involvement. Type 1 GD (GD1) is the most frequent and it is characterized by its extreme heterogeneity which includes asymptomatic or more severe presentations. GD1 is distinguished by the absence of primary neurological involvement. The accumulation of glucosylceramide in visceral organs generates splenomegaly in $90 \%$ of patients and hepatomegaly in between $60-80 \%$ of those affected with the disease. Consequently, the decrease of the concentration of red blood cells and thrombocytopenia is frequent. likewise, about $50 \%$ of the patients suffer from chronic fatigue which often impacts their daily activity and their socio-professional activities. [11] Otherwise, the Type 2 GD (GD2) and Type 3 GD (GD3) are associated with neurological affectation that can become serious.

Traditionally, the diagnosis has been made through a clinical examination accompanied by a biochemical study measuring the levels of the activity of the enzyme B-Glucocerebrosidase in peripheral blood leukocytes.[12] However, enzymatic studies do not allow the distinction between healthy individuals and carriers; it is for this reason that nowadays an analysis of specific variants to the gene of interest is being carried out, or adopting the use of molecular techniques such as the sequencing of the complete exome for effects of phenotype-genotype associations and broader knowledge about the variants associated to the disease.[12-13] As for treatment, therapeutic approaches such as Enzyme Replacement Therapy (ERT) offers a secure therapy for GD patients with 3 different available enzymes worldwide: Imiglucerase (Sanofi-Genzyme) Velaglucerasa alfa (Takeda pharmaceuticals) and taliglucerase alfa, which is plant-cell derived that can be produced on a large scale at lower cost expressed.[3]

The present study aims to characterize molecularly the variants of the GBA gene in patients from Southwest of Colombia to recognize which ones are commonly circulating and to understand the genotypic effect on the phenotype of these patients.

\section{Method}

\section{Type of Study}

An observational, descriptive, transversal, and prospective study was conducted, in which 19 patients from southwest of Colombia, diagnosed clinically and enzymatically with GD accepted to participate in the study, with prior informed consent. An analysis of the medical records of patients with GD was previously carried out.

\section{Inclusion Criteria}

We included patients with GD of all ages who had a result of molecular diagnosis. Patients and relatives who signed the respective consent and assent informed.

\section{Exclusion Criteria}

Patients without molecular diagnosis of Gaucher disease

\section{Sequencing}

To identify the variants in each patient, peripheral blood samples were previously taken and sent to analyze. DNA was isolated using the standard salting-out method and quantified using a QIAxpert (Qiagen). The DNA samples were purified using The Genomic DNA Clean \& Concentrator ${ }^{\mathrm{m}}-25$ (DCC $\left.^{\mathrm{mm}}\right)$ Kit and were stored at $-20^{\circ} \mathrm{C}$ until investigated. The $G B A$ gene was analyzed to polymerase chain reaction amplification (PCR) and sequencing of the entire coding region as well as the highly conserved (exon-intron) junctions in genomic DNA according to the CENTOGENE laboratory indications. The GBA gene reference sequence is: NM_000157.3. Each amplified product was analyzed by a standard fluorescent sequencing protocol in both the forward and reverse direction. Full sequencing analysis of the $G B A$ gene detects approximately $99 \%$ of disease-causing mutations (http://www.ncbi.nlm.nih.gov/sites/GeneTests). Only the coding regions of the $G B A$ gene and immediate flanking intron sequences were examined. Changes in the promoter region, farther into the intronic regions, or in other noncoding regions of the gene would not be identified. A minimum coverage of $>20 \mathrm{x}$ was obtained for each amplicon. 


\section{Analysis of Data}

The analysis of the obtained sequence was performed with the bioinformatic program SeqScape Software v3 (TermoFisher Scientific) and the annotation of the obtained variants was developed with the program Alamut VisualTM (Interactive Biosoftware). The identification of the variants of interest was carried out concerning the reference genome (hg19).

The used reference databases were the population databases dbSNP, 1000genomes, EXAC and gnomAD, the clinical databases Human Gene Mutation Database (HGMD version 2019.4) ClinVar and LOVD, specific databases of the disease, if applicable, and some own Reference Laboratory Genetics databases.

\section{Bioinformatic Analysis}

With the bioinformatic programs Mutation Taster, SIFT, Human Splicing Finder, UMD-Predictor, Provean, SNPeffect, SNPs3D and PolyPhen2, bioinformatic analysis was carried out to evaluate the possible impact of the variants of interest on the protein structure and functionality and to predict the impact on the protein of amino acid substitutions. The classification of the variants was made based on the recommendations of the American College of Medical Genetics and Genomics (ACMG).[10]

\section{Interaction Network}

Finally, a gene interaction network of the $G B A$ gene was carried out through STICH 5 program to determine close associations with other genes that allowed determining physical interactions, levels of co-expression and use of medications related to the indicated treatment.

\section{Bioethical Aspects}

The Ethics committee of the Universidad Santiago de Cali approved the study, and it was performed in accordance with the tenets of the World Medical Association Declaration of Helsinki. the level of this research has been categorized with a minimal risk since it presents a low risk of physical harm to the participants as it is a retrospective study of review results.

Confidentiality of data: The authors state that no patient data appear in this article, and they have followed the protocols of their center of work.

Right to privacy and informed consent: The authors declare that there is no personal data of patients in this article; it was obtained the corresponding consent and informed assent for the patients' samples processing, and confidential data use by the patient's legal representative.

\section{Results}

The patients included in the study presented hepatomegaly (52,6\%), splenomegaly $(73,6 \%)$, anemia $(57,8 \%)$, and thrombocytopenia $(42,1 \%)$ with or without any bone abnormality. Table 1 covers the clinical details of the patients. Adult patients never had any symptoms in childhood. The review of the clinical history of each patient, allowed to find specifically that: Patient 3 presented a report of hepatosplenomegaly and grade III hepatic steatosis; In addition, a limitation of gait was confirmed, associated with left hemiparesis, retractions in general and appearance of bone necrosis. This patient had a slight delay in neurodevelopment and does not have sphincter control.

Table 1. Clinical details profile of patients from Southwest of Colombia with Gaucher disease.

\begin{tabular}{|c|c|c|c|c|c|c|c|c|c|c|c|c|c|c|c|c|c|c|c|}
\hline Patient ID & P1 & P2 & P3 & P4 & P5 & P6 & P7 & P8 & P9 & P10 & P11 & P12 & P13 & P14 & P15 & P16 & P17 & P18 & P19 \\
\hline Age & 4 & 3 & 6 & 15 & 45 & 23 & 42 & 56 & 52 & 37 & 22 & 55 & 59 & 58 & 25 & 50 & 71 & 17 & 36 \\
\hline Sex & $M$ & M & $\mathrm{F}$ & $\mathrm{F}$ & $M$ & $M$ & M & $M$ & $\mathrm{~F}$ & M & $\mathrm{F}$ & $\mathrm{F}$ & M & M & $M$ & $\mathrm{~F}$ & $\mathrm{~F}$ & $M$ & $\mathrm{~F}$ \\
\hline $\begin{array}{l}\text { Decreased enzyme } \\
\text { activity }\end{array}$ & $x$ & $x$ & $x$ & $x$ & $x$ & $x$ & $x$ & $x$ & $x$ & $x$ & $x$ & $x$ & $x$ & $x$ & $x$ & $x$ & $x$ & $x$ & $x$ \\
\hline \multicolumn{20}{|l|}{ Generalized Weakness } \\
\hline Splenomegaly & $x$ & $x$ & $x$ & $x$ & & & & $x$ & $x$ & $x$ & $x$ & $x$ & $x$ & $x$ & & $x$ & & $x$ & $x$ \\
\hline Hepatomegaly & $x$ & & $x$ & $x$ & & & & & $x$ & $x$ & $x$ & $x$ & & & & $x$ & & $x$ & $x$ \\
\hline \multicolumn{20}{|l|}{$\begin{array}{l}\text { Hematolical } \\
\text { abnormalities }\end{array}$} \\
\hline Anemia & $x$ & & $x$ & $x$ & & & & & $x$ & $x$ & $x$ & & $x$ & $x$ & $x$ & & $x$ & $x$ & \\
\hline Thrombocytopenia & & & $x$ & & & $x$ & & $x$ & $x$ & $x$ & $x$ & $x$ & & & & & & $x$ & \\
\hline Cytopenia & $x$ & & $x$ & & & & & & & & & & & & & & & & \\
\hline Bone marrow analysis & & $x$ & $x$ & $x$ & & & & & & $x$ & $x$ & & & & & & & $x$ & \\
\hline \multicolumn{20}{|l|}{ Bone abnormalities } \\
\hline Difficulty in walking & & & $x$ & & & & & & & & & $x$ & & & & & $x$ & & $x$ \\
\hline Avascular necrosis & & & $x$ & & & & & $x$ & & & & $x$ & & & & & & & $x$ \\
\hline $\begin{array}{l}\text { Enzyme Replacement } \\
\text { Therapy }\end{array}$ & $x$ & $x$ & $x$ & $x$ & $x$ & $x$ & $x$ & $x$ & $x$ & $x$ & $x$ & & $x$ & $x$ & $x$ & $x$ & & $x$ & $x$ \\
\hline
\end{tabular}

Abbreviation : F Female, M Male 
Patients 4,5,6 and 7 are part of the same family group, with a family history of anemia and bone necrosis. Patients 5 and 7 presented decreased enzyme activity values, without any associated symptoms. Patient 4 was diagnosed with bicytopenia, perivascular dermatitis, erythema nodosum in the lower limbs and severe dilatation of the blood vessels of the lower limbs. The total abdominal ultrasound performed on patient 6 revealed a liver of normal size, shape, and contour; however, a thick 9.0 $\mathrm{mm}$ calcification was observed in segment $\mathrm{V}$ of the right lobe of the liver.

Patient 17 presented symptoms consisting of severe anemia after the age of 60 , the enzymatic tests presented very low concentration values, for which the diagnosis was made.

Patients 10-11, 14-15 and 17-18 make up three different family groups.

The pelvis MRI report of the patient 19 revealed hyperintensities over the upper shaft of the right femur along with changes in pelvic floor muscles.

Only two patients did not receive ERT at the time of the study due to technical difficulties of the health service.

In the molecular diagnosis of 19 patients clinically and enzymatically diagnosed with GD, 11 male patients (57.8\%) were found with an age range between 4 and 71 years. Additionally, it was possible to determine the affected exons, nucleotide changes, protein changes and respective zygosity (14/19 homozygous;
4/19 heterozygous compounds; $1 / 19$ heterozygous) in each of the patients (Table 2).

A single variant (p.Arg535His) in a heterozygous status was found in an affected patient. Patients P1, P4, P5, P6, P8, P12, P18 were found to carry a homozygous copy of c.1226A>G p.Asn409Ser variant in exon 9 of GBA gene (Table 2).

Overall, we have identified 23 alleles with GBA variants. From the seven variants found, specifically the p.Asn409Ser (10/23 alleles), the p.Leu483Pro (3/23 alleles), and the p.Lys237Glu (3/23 alleles) are present in $69.5 \%$ of the alleles.

About the genotype frequency, the homozygous variant p.Asn409Ser / p.Asn409Ser was found in 36\%, followed by the homozygous variants p.Leu483Pro / p.Leu483Pro; p.Lys237Glu / p.Lys237Glu; p.Glu365Lys / p.Glu365Lys each found with a frequency of $10 \%$ (Table 3).

The bioinformatic analysis of all the variants found through the bioinformatics software described a pathogenic clinical significance in all the cases and established the functional effects of the variants identified; these variants have been identified in previously published data (dbSNP code).

The gene interaction network allowed observing close associations between the GBA gene and the PSAP, SCARB2 and $L A M P 2$ genes; all with functions related to vacuolar locations, lysosomal and vacuolar lumen associated instructions, vacuolar and lysosomal membranes (Figure 1).

Table 2. Molecular characterization of the variants in the GBA gene found in 19 patients affected with Gaucher disease.

\begin{tabular}{|c|c|c|c|c|c|c|}
\hline \# Patient & Exon affected & $\begin{array}{c}\text { nucleotide } \\
\text { change }\end{array}$ & protein change & dbSNP & Zygosity & $\begin{array}{c}\text { Bioinformatic } \\
\text { Analysis }\end{array}$ \\
\hline 1 & 9 & c. $1226 \mathrm{~A}>\mathrm{G}$ & p.Asn409Ser & rs76763715 & Homozygous & pathogenic \\
\hline 2 & 10 & c. $1448 T>C$ & p.Leu483Pro & rs421016 & Homozygous & pathogenic \\
\hline 3 & 9 & c. $1279 \mathrm{G}>\mathrm{A}$ & p.Glu427Lys & rs149171124 & Homozygous & pathogenic \\
\hline 4 & 9 & c. $1226 \mathrm{~A}>\mathrm{G}$ & p.Asn409Ser & rs76763715 & Homozygous & pathogenic \\
\hline 5 & 9 & c. $1226 \mathrm{~A}>\mathrm{G}$ & p.Asn409Ser & rs76763715 & Homozygous & pathogenic \\
\hline 6 & 9 & c. $1226 \mathrm{~A}>\mathrm{G}$ & p.Asn409Ser & rs76763715 & Homozygous & pathogenic \\
\hline 7 & 10 & c. $1448 T>C$ & p.Leu483Pro & rs421016 & Homozygous & pathogenic \\
\hline 8 & 9 & c. $1226 \mathrm{~A}>\mathrm{G}$ & p.Asn409Ser & rs76763715 & Homozygous & pathogenic \\
\hline 9 & 6 & c.709A $>G$ & p.Lys237Glu & rs77340931 & Homozygous & pathogenic \\
\hline 10 & 9 & $\begin{array}{l}\text { c.595_596del } \\
\text { c. } 1226 \mathrm{~A}>\mathrm{G}\end{array}$ & $\begin{array}{l}\text { p.Leu199Aspfs*62 } \\
\text { p.Asn409Ser }\end{array}$ & $\begin{array}{l}\text { rs74971446 } \\
\text { rs76763715 }\end{array}$ & Heterozygous & pathogenic \\
\hline 11 & 129 & $\begin{array}{l}\text { c.595_596del } \\
\text { c.1226A>G }\end{array}$ & $\begin{array}{l}\text { p.Leu199Aspfs*62 } \\
\text { p.Asn409Ser }\end{array}$ & $\begin{array}{l}\text { rs74971446 } \\
\text { rs76763715 }\end{array}$ & Homozygous & pathogenic \\
\hline 12 & 9 & c. $1226 \mathrm{~A}>\mathrm{G}$ & p.Asn409Ser & rs76763715 & Homozygous & pathogenic \\
\hline 13 & 9 & c. $1093 \mathrm{G}>\mathrm{A}$ & p.Glu365Lys & rs2230288 & Homozygous & pathogenic \\
\hline 14 & 9 & c. $1093 \mathrm{G}>\mathrm{A}$ & p.Glu365Lys & rs 2230288 & Homozygous & pathogenic \\
\hline 15 & 910 & $\begin{array}{l}\text { c. } 1226 \mathrm{~A}>\mathrm{G} \\
\text { c. } 1448 T>C\end{array}$ & $\begin{array}{l}\text { p.Asn409Ser } \\
\text { p.Leu483Pro }\end{array}$ & $\begin{array}{l}\text { rs76763715 } \\
\text { rs421016 }\end{array}$ & Heterozygous & pathogenic \\
\hline 16 & $N R$ & c. $1604 \mathrm{G}>\mathrm{A}$ & p.Arg535His & rs75822236 & Heterozygous & pathogenic \\
\hline 17 & 712 & $\begin{array}{l}\text { c. } 709 A>G \\
\text { c. } 1604 G>A\end{array}$ & $\begin{array}{l}\text { p.Lys237Glu } \\
\text { p.Arg535His }\end{array}$ & $\begin{array}{l}\text { rs77340931 } \\
\text { rs75822236 }\end{array}$ & Heterozygous & pathogenic \\
\hline 18 & 9 & c. $1226 \mathrm{~A}>\mathrm{G}$ & p.Asn409Ser & rs76763715 & Homozygous & pathogenic \\
\hline 19 & 7 & c.709A $>G$ & p.Lys237Glu & rs77340931 & Homozygous & pathogenic \\
\hline
\end{tabular}


Table 3. Genotypic frequency found in patients diagnosed with GD.

\begin{tabular}{ll}
\hline Genotype & Frequency \\
\hline p.Asn409Ser/p.Asn409Ser & $0.36(7 / 19)$ \\
p.Leu483Pro/p.Leu483Pro & $0.10(2 / 19)$ \\
p.Glu427Lys/p.Glu427Lys & $0.05(1 / 19)$ \\
p.Lys237Glu/p.Lys237Glu & $0.10(2 / 19)$ \\
p.Leu199Aspfs*62 / p.Asn409Ser & $0.10(2 / 19)$ \\
p.Glu365Lys/p.Glu365Lys & $0.10(2 / 19)$ \\
p.Asn409Ser/p.Leu483Pro & $0.05(1 / 19)$ \\
p.Arg535His & $0.05(1 / 19)$ \\
p.Lys237Glu/ p.Arg535His & $0.05(1 / 19)$ \\
\hline
\end{tabular}

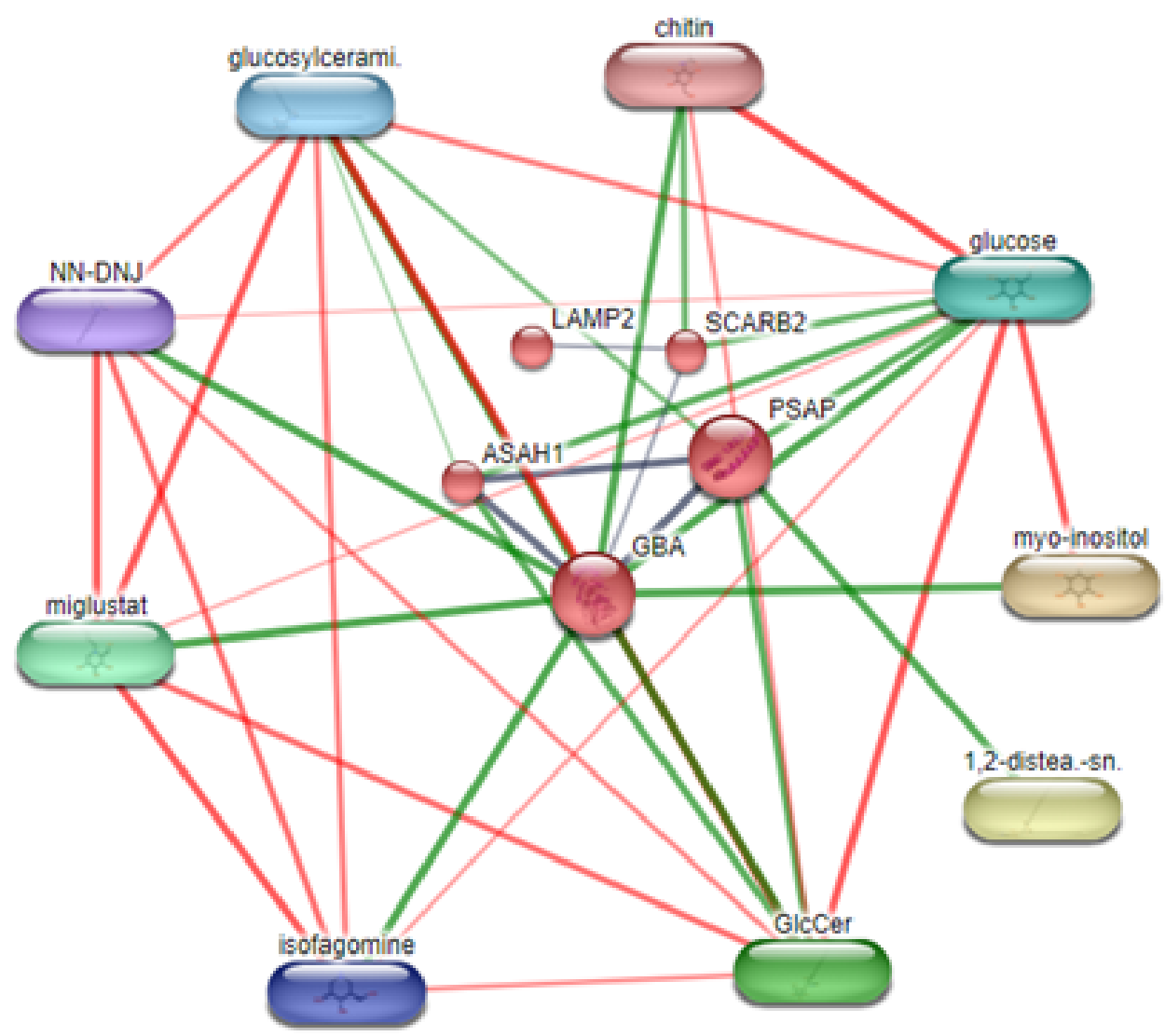

Figure 1. Interaction network between the GBA gene and associated genes [Elaboration: Own source, STICH 5].

\section{Discussion}

Patients enrolled in this study exhibited clinical presentations like hepatosplenomegaly, with anemia, and bone manifestations in some of them, this generally occurs in GD with a prevalence in more than $90 \%$ of cases.[10] Hepatosplenomegaly occurs when Gaucher cells are accumulated in the liver and spleen, resulting in an abnormal increase in their size. In some cases, spleen size may be as much as 15 times larger than normal. This enlargement generally causes abdominal distension and pain, this being an alarm sign to identify the disease.
With respect to ERT, the median age of initiation of treatment is earlier in Latin America than in the rest of the world (16 vs. 27 years). In $77 \%$ of the patients, treatment started below 17 years old, according to the ICGG data[14]; This therapy has successfully modified the phenotype of patients with GD, which is why it constitutes the treatment of choice, with a positive impact on the quality of life of these patients. In the case of the patients in the present study, only 2 patients do not have ERT to date due to delays in the health system, however, all continue to be monitored and followed up by the treating physicians.[14-15] 
J. inborn errors metab. screen.

On the other hand, the different variants reported in the GBA gene sometimes make the molecular study of the patients difficult; nevertheless, the molecular analysis of the GBA gene in the present work allowed identifying the genotype of 19 patients of the Colombian southwest with GD.

Bioinformatic analysis using in-silico technology that predicts the probability of damage generated by an amino acid SNP in the structure and function of a protein, in each of the variants found, demonstrated a possibility of complete damage to the GBA protein in each one of the cases. These tools predict whether an amino acid substitution affects protein function based on sequence homology and the physical properties of amino acids, which indicates through experiment means that changes in the protein have a potential effect that generates damage to functions.

The different variants may be more represented ethnic groups, as well as phenotypes. The first variant reported in GD was p. Leu483Pro.[16] The most common one identified in Romanian patients is p. Asn409Ser.[19] The most common among $\mathrm{AJ}$ patients is c.1226A $<\mathrm{G}$ (p.Asn409Ser) followed by c.84dupG (84GG), which is more rare.[17] The change c.115 + 1G> A (IVS2 + 1), c.1504C> T p. (Arg463Cys) and c.1604G> A (p.Arg496His) is commonly found in AJ patients with GD1. In contrast, the variant $\mathrm{p}$.Asn409Ser is rarely found among Chinese and Japanese patients.[18]

It has been found that different variants may lead to different phenotypes of GD. The variant p.Asn409Ser is only associated with GD1, and it seems to be protective for the development of the characteristic neurological affectation of GD2 and GD3. Patients who are homozygous for the p.Asn409Ser variant may also remain asymptomatic for the disease. In the present study, the patients who were homozygous for this variant shared some symptoms such as splenomegaly and anemia, however, according to the degree of the disease, some showed more affection than others, some being asymptomatic until adulthood, so an exact phenotype-genotype correlation is not possible. $[17,18]$

On the other hand, the variant p.Leu483Pro is generally associated with GD2 and GD3 even when it appears in a compound heterozygous state.[19] The homozygous variant p.Leu483Pro / Leu483Pro without recombinant alleles can be associated with very severe phenotypes, but also milder ones. Therefore, no specific phenotype-genotype relationship has been defined thus far.[20] Giraldo et al. (2000) showed the high prevalence of the p.L483P in Spanish patients which suggested Hispanic ancestry among patients with this mutation.[21] According to studies conducted by Wilches et al. (2006), this is the most frequent mutation, among other GBA mutations, in the Colombian population, more specifically in the Cundinamarca and Boyacá regions.[22] In our case, the two patients who presented this variant in a homozygous state showed a mild phenotype; one of them presented alterations in the bones and splenomegaly, while the other did not present any clear symptoms and only enzymatic tests with decreased values were found.
Pomponio et al. (2005) described GBA mutations from 25 Colombian patients with GD. These mutations were c.595_596delCT, c.898delG and c.1255G > C in exons 6, 7, and 9 of the GBA gene, respectively. In addition, they found a double mutation (p.Leu483Pro + p.Glu427Lys) in three patients with GD type I, they showed that $63.1 \%$ of the mutated alleles corresponded to the variants p.Asn409Ser and p.Leu483Pro, with $36.9 \%$ of alleles in which a variant has been detected with less frequency [23]. In our case, $43 \%$ of the mutated alleles corresponded to the variant p.Asn409Ser and 13\% to p.Leu483Pro, confirming that both variants continue to present a high circulation among affected patients (56\%).

Considering the literature on the pathogenicity of the p.Glu427Lys variant (also known as E388K), contradictory results have been found since it has been identified in the control population and previous studies conducted on patients with GD. According to the bioinformatic tools used in the present study, this variant was reported in one case and characterized with significance as a pathogenic clinic; likewise, in investigations carried out by Kumar et al. (2012), this variant in the GBA gene has been associated with the risk of Parkinson's Disease (PD).[24-26]

On the other hand, the variant p. Lys237Glu has been reported in a low prevalence associated with GD. In this study, two homozygous patients were found for this variant. The research carried out by Goker-Alpan et al. (2003) reported the presence of this variant in a 16-month-old female patient with a molecular diagnosis of GD2, who presents phenotype p. Lys237Glu/ Lys237Glu; the research described the patient with severe organomegaly, horizontal supranuclear paralysis, cherry-red spots in the eyes, and myoclonic seizures refractory to conventional antiepileptic therapy. High-dose ERT was used, and the efficacy of ERT began soon after the diagnosis was limited to the correction of hematological parameters.[27]

The variant $\mathrm{p}$.Leu199Aspfs ${ }^{*} 62$ / p.Asn409Ser was found in two of the patients in this study. Pomponio et al. (2005) previously reported new GBA variants in 25 Colombian patients with GD. These variants were c.595_596delCT (p.Leu199Aspfs ${ }^{*} 62$ ), c.898delG and c.1255G>C in exons 6, 7, and 9 of the GBA gene, respectively; the presence of these variants was not entirely unexpected due to the unique genetic background of the Colombian population, as it has been observed in other diseases such as MPS IVA.[23,24] Specifically, their studies reported the variant p. Leu199Aspfs ${ }^{\star} 62$ / p.Asn409Ser in a patient from Cundinamarca, who presented splenomegaly, hepatomegaly, anemia, and thrombocytopenia.[29]

Regarding the homozygous variant p. Glu365Lys, it was found in two patients affected with GD in this study. Moore et al. (2019) reported the variant in a patient PD and dementia with Lewy bodies (LB). It should be pointed that although this patient met the biochemical (low GCas activities) and genetic criteria to be diagnosed with GD, she was clinically and pathologically referred to as affected by PD, so future studies will be necessary to determine the correct phenotype-genotype association.[30] 
The variant p.Arg535His was found in this study in a homozygous and heterozygous compound state in 2 patients; Siebert et al. (2012) reported this variant in $1 / 48$ patients with GD who presented low GCase activity in leukocytes or fibroblasts or both and at least one allele causing disease not identified after the detection of common variants (N370S, L444P, 84insG, and IVS2p1G> A).[31] It was also reported by Ruskey et al. (2018) in 9/375 with moderate PD and phenotypic association of GD1.[32] If the clinical presentation of the patient is due to this mutation in state heterozygous, raises the paradigm of a behavior with inheritance mechanism dominant or the presence of other alterations in the gene allele, requiring further investigation biochemical and molecular tests for genotype-phenotypic correlation and genetic counseling suitable.

Finally, from the GBA-based gene interaction network, a close relationship between some of them can be determined, maintaining a high genetic interaction, which could possibly lead to specific phenotypes related to the observed phenotype and the clinical manifestations that they occur in patients with GD.[33] These networks make it possible to demonstrate the natural function of each of the genes, necessary for the development of biological processes such as catabolism and lipid metabolism. This is how the close interaction of the GBA gene with nearby genes such as PSAP, LAMP2 and SCARB2 generates that a specific damage in one of these genes alters the activity of the others, giving rise to side effects in the organism.[34]

It has been widely discussed that the main activator of $\beta$-glucocerebrosidase, saposin $C$, is encoded by the prosaposin gene (PSAP) located at the 10q21-22 locus; Saposin C is an established activator for the hydrolysis of glucosylceramide by GCase in lysosomes, however, the mechanism by which Saposin C promotes lysosomal hydrolysis is not fully understood.[34]

Nowadays it is recognized that PSAP conditions cause alterations in GBA activity, giving rise to typical signs such as hepatosplenomegaly and neurological compromise, being related to GD type 2 [35], which has led to the search for modifiers of disease that may alter the GD phenotype. In humans, saposin $\mathrm{C}$ deficiency due to mutations in PSAP results in an GD-like phenotype, despite having normal in vitro glucocerebrosidase activity values. Saposin C deficiency has also been shown to modify the phenotype in murine GD models.[34]

Transcription factors responsible for the differential expression of the GBA gene in specific tissues include proteins such as OCTA, AP-1, PEA3, and CAAT. Deficiencies in the genes encoding lysosome-associated membrane proteins (LAMP1 and LAMP-2) and involved in the intracellular transport of glucosylcerebrosidase from the endoplasmic reticulum to lysosomes may also be present in patients with GD.[36-37] Studies by Kurzawa-Akanbi et al. (2012) reported that carriers of pathogenic GBA mutations demonstrated a significantly reduced level of GCase protein and enzymatic activity and retention of glucocerebrosidase isoforms within the endoplasmic reticulum (ER), which was associated with an increased expression of the lysosomal markers LAMP1 and LAMP2, although the expression of ATP13A2 and cathepsin D was reduced, together with the decrease in cathepsin D activity, so understanding the way in which genes close to GBA interact will allow a better understanding of phenotypic and genotypic characteristics in affected patients.[38]

\section{Conclusions}

The given study reports 19 patients with GD with all mutations clustered in exon 8 and 10. Also, the study contributes seven variants to the spectrum of $G B A$ gene mutations along with providing knowledge about the prevalence of most common $G B A$ gene mutation c.1226A $>$ G p.Asn409Ser in a general Colombian population. This can help in maintaining population health quotient by doing pre-marital counseling and hence minimizing the disease burden amongst the population. In view of milder clinical manifestation during adult GD, differential diagnosis of GD needs to be suspected in patients with unexplained anemia, pancytopenia and/or hepato/splenomegaly.

Research, diagnosis, and treatment of GD in Colombia holds a great potential given the amount of studies done for this disorder. Knowledge about this disease opens an opportunity develop novel treatments, to improve existing ones, and to strengthen the basic and clinical research of GD in our country.

\section{Declaration of Conflicting Interests}

The authors declare no conflict of interest. Project funded by the DGI-03-2020 call: Applied research projects, Universidad Santiago de Cali. Special thanks to the participating patients, the Congenital Metabolism Diseases Research Group, Colombian Interest Group on Gaucher's Disease (GICEG), Universidad Santiago de Cali.

\section{References}

1. Nguyen Y, Stirnemann J, Belmatoug N. Gaucher disease': A review. Rev Med Interne. 2019;40(5):313-322. doi:10.1016/j. revmed.2018.11.012

2. Sun A. Lysosomal storage disease overview. Ann Transl Med. 2018;6(24):476. doi:10.21037/atm.2018.11.39

3. Andrade-Campos MM, de Frutos LL, Cebolla JJ, et al. Identification of risk features for complication in Gaucher's disease patients: a machine learning analysis of the Spanish registry of Gaucher disease. Orphanet J Rare Dis. 2020;15:256. doi:10.1186/s13023-020-01520-7

4. Mehta A. Epidemiology and natural history of Gaucher's disease. Eur J Intern Med. 2006;17(Suppl):s2-s5. doi:10.1016/j.ejim.2006.07.005

5. Nalysnyk L, Rotella P., Simeone J., Hamed A., Weinreb N. Gaucher disease epidemiology and natural history: a comprehensive review of the literature. Hematology. 2017;22(2):65-73. doi:10.1080/10245332.2016.1240391 
6. Orphanet. Enfermedad de Gaucher. https://www.orpha.net/ consor/cgi-bin/oc_exp.php?lng=es\&expert=355. Accessed March 12, 2019.

7. ADRES. Análisis de los recobros correspondientes a los principios activos imiglucerasa, miglustato, velaglucerasa y taliglucerasa medicamentos para la enfermedad de Gaucher. Published 2017. https://www.adres.gov.co/ la-entidad/publicaciones/post/5993/analisis-de-losrecobros-correspondientes-a-los-principios-activosimiglucerasa-miglustato-velaglucerasa-y-taliglucerasamedicamentos-para-la-enfermedad-de-gaucher. Accessed March 12, 2019.

8. Uribe A, Giugliani R. Selective screening for lysosomal storage diseases with dried blood spots collected on filter paper in 4,700 high-risk colombian subjects. JIMD Rep. 213;11:107-116. doi:10.1007/8904_2013_229

9. Puentes-Tellez MA, Lerma-Barbosa PA, Garzón-Jaramillo $\mathrm{RG}$, et al. A perspective on research, diagnosis, and management of lysosomal storage disorders in Colombia. Heliyon. 2020;6(3):e03635. doi:10.1016/j.heliyon.2020. $\mathrm{e} 03635$

10. Sheth J, Pancholi D, Mistri M, Nath P, Ankleshwaria C, Bhavsar R. Biochemical and molecular characterization of adult patients with type I Gaucher disease and carrier frequency analysis of Leu444Pro - a common Gaucher disease mutation in India. BMC Med Genet. 2018;19(1):178. doi:10.1186/s12881-018-0687-5

11. Von Rossum A, Holsopple M. Enzyme Replacement or Substrate Reduction? A Review of Gaucher Disease Treatment Options. Hosp Pharm. 2016; 51(7): 553-563. doi:10.1310/hpj5107-553

12. Lavaut K, Núñez A, Nordet I, et al. Aspectos clínicos, bioquímicos, moleculares y tratamiento de 2 pacientes con enfermedad de Gaucher. Rev Cubana Hematol Inmunol Hemoter. 2010;26(1):54

13. Colquicocha-Murillo M, Cucho-Jurado J, EyzaguirreZapata R, et al. Guía para diagnóstico y tratamiento de la Enfermedad de Gaucher. Rev Med Hered. 2015;26(2):103121. http://www.scielo.org.pe/pdf/rmh/v26n2/a07v26n2. pdf

14. Bennett LL, Mohan D. Gaucher disease and its treatment options. Ann Pharmacother. 2013;47(9):1182-1193. doi:10.1177/1060028013500469

15. Drelichman G, Linares A, Villalobos J, et al. Enfermedad de Gaucher en Latinoamérica: un informe del Registro Internacional y del Grupo Latinoamericano para la Enfermedad de Gaucher. Medicina (B. Aires). 2012;72(4):273-282

16. Tsuji S, Choudary PV, Martin BM, et al. A Mutation in the Human Glucocerebrosidase Gene in Neuronopathic Gaucher's Disease. N Engl J Med. 1987;316(10): 570-575. doi:10.1056/NEJM198703053161002
17. Riboldi GM, Di Fonzo AB. GBA, Gaucher disease, and parkinson's disease: from genetic to clinic to new therapeutic approaches. Cells. 2019;8(4):364. doi:10.3390/ cells8040364

18. Hruska KS, LaMarca ME, Scott CR, Sidransky E. Gaucher disease: mutation and polymorphism spectrum in the glucocerebrosidase gene (GBA). Hum Mutat. 2006;29(5):567-588. doi:10.1002/humu.20676

19. Stirnemann J, Belmatoug N, Camou F, et al. A review of gaucher disease pathophysiology, clinical presentation and treatments. Int J Mol Sci. 2017;18(2):441. doi:10.3390/ ijms 18020441

20. Goker-Alpan O, Hruska K, Orvisky E, Kishanani P, Stubblefield BK, Schiffmann R, Sidransky E. Divergent phenotypes in Gaucher disease implicate the role of modifiers. J Med Genet. 2005;42(6):e37. doi:10.1136/ jmg.2004.028019

21. Giraldo P, Pocovi M, Perez-Calvo J, Rubio-Felix D, Giralt M. Report of the Spanish Gaucher's disease registry: clinical and genetic characteristics. Haematologica. 2000;85(8):792799. https://haematologica.org/article/view/1757

22. Wilches R, Vega H, Echeverri O, Barrera LA. Los haplotipos colombianos de la mutación N370S causante de la enfermedad de Gaucher pueden provenir de un haplotipo ancestral común. Biomedica. 2006;26(3):434-441

23. Pomponio RJ, Cabrera-Salazar MA, Echeverri OY, Miller G, Barrera LA. Guacher disease in Colombia: mutation identification and comparison to other Hispanic populations. Mol Genet Metab. 2005;86:466472. doi:10.1016/j.ymgme.2005.07.026

24. Kumar K, Ramirez A, Gobel A, et al. Glucocerebrosidase mutations in a Serbian Parkinson's disease population. Eur J Neurol. 2013;20(2):402-405. doi:10.1111/j.14681331.2012.03817.x

25. Liu G, Boot B, Locascio JJ, et al. Specifically neuropathic Gaucher's mutations accelerate cognitive decline in Parkinson's. Ann Neurol. 2016;80(5):674-685. doi:10.1002/ ana. 24781

26. Paciottia S, Persichettia E, Pagliardini S, et al. First pilot newborn screening for four lysosomal storage diseases in an Italian region: Identification and analysis of a putative causative mutation in the GBA gene. Clin Chim Acta. 2012;413(23-24):1827-1831. doi:10.1016/j.cca.2012.07.011

27. Goker-Alpan O, Schiffmann R, Park J, Stubblefield B, Tayebi $\mathrm{N}$, Sidransky E. Phenotypic continuum in neuronopathic gaucher disease: an intermediate phenotype between type 2 and type 3. J Pediatr. 2003;143(2):273-276. doi:10.1067/ S0022-3476(03)00302-0

28. Giraldo- Moreno LJ, Arturo-Terranova D, Satizabal-Soto JM. Otorhinolaryngological Findings in Patients from Southwestern Colombia with Clinical, Enzymatic and 
Molecular Diagnosis of Mucopolysaccharidosis II, IV-A and VI. J Inborn Errors Metab. Screen. 2020;8:e20190006. doi:10.1590/2326-4594-jiems-2019-0006

29. Kato Z, Fukuda S, Tomatsu S, Vega H, Yasunaga T, Yamagishi A. A novel common missense mutation G301C in the N-acetylgalactosamine-6-sulfate sulfatase gene in mucopolysaccharidosis IVA. Hum Genet. 1997;101(1):97101. doi:10.1007/s004390050594

30. Moors TE, Paciotti S, Ingrassia A. et al. Characterization of Brain Lysosomal Activities in GBA-Related and Sporadic Parkinson's Disease and Dementia with Lewy Bodies. Mol Neurobiol. 2019;56:1344-1355. doi:10.1007/s12035-0181090-0

31. Siebert M, Bock H, Michelin-Tirelli K, Coelho J, Giugliani R, Saraiva-Pereira ML. Novel mutations in the glucocerebrosidase gene of brazilian patients with Gaucher disease.JIMD Rep.2013;9:7-16.doi:10.1007/8904_2012_174

32. Ruskey JA, Greenbaum L, Roncière L, et al. Gan-Or, Increased yield of full GBA sequencing in Ashkenazi Jews with Parkinson's disease. Eur J Med Genet. 2019;62(1):6569. doi:10.1016/j.ejmg.2018.05.005
33. Sevimoglu T, Yalcin K. The role of protein interaction networks in systems biomedicine. Comput Struct Biotechnol J. 2014;11(18):22-27. doi:10.1016/j.csbj.2014.08.008

34. Tamargo RJ, Velayati A, Goldin E, Sidransky E. The role of saposin C in Gaucher disease. Mol Genet Metab. 2012;106(3):257-263. doi:10.1016/j.ymgme.2012.04.024

35. Acanda A. Aspectos bioquímicos, genéticos y comorbilidades de la enfermedad de Gaucher, diagnóstico molecular en Cuba. Rev Cubana Genet Comunit. 2012;6(1):8-19

36. Schnabel D, Schroder M, Sandhoff K. Mutation in the sphingolipid activator protein 2 in a patient with a variant of Gaucher disease. FEBS Lett. 1991;284(1):5759. doi:10.1016/0014-5793(91)80760-Z

37. Martinez-Arias R, Comas D, Mateu E, Bertranpetit J. Glucocerebrosidase pseudogene variation and Gaucher disease: recognizing pseudogene tracts in GBA alleles. Hum Mutat. 2001;17(3):191-198. doi:10.1002/humu.4

38. Kurzawa-Akanbi M, Hanson PS, Blain PG, Lett DJ, McKeith IG, Chinnery PF, Morris CM. Glucocerebrosidase mutations alter the endoplasmic reticulum and lysosomes in Lewy body disease. J Neurochem. 2012;123(2):298-309. doi:10.1111/j.1471-4159.2012.07879.x 